\title{
Associations Between Body Mass Index and Suicide in the Veterans Affairs Health System
}

\author{
John F. McCarthy ${ }^{1,2,3,4}$, Mark A. Ilgen ${ }^{1,2,3,4}$, Karen Austin ${ }^{1,3}$, Frederic C. Blow ${ }^{1,2,3,4}$ and Ira R. Katz ${ }^{1}$
}

Objectives: Associations between BMI and suicide risks and methods for individuals receiving care in the Veterans Health Administration (VHA) health system were evaluated.

Design and Methods: For 4,005,640 patients in fiscal years 2001-2002, multivariable survival analyses assessed associations between BMI and suicide, through FY2009. Covariates included demographics, psychiatric, and nonpsychiatric diagnoses, receipt of VHA mental health encounters, and regional network. Among suicide decedents, multivariable Generalized Estimating Equations (GEE) regression examined associations between BMI and suicide method.

Results: $1.3 \%$ of patients were underweight, $24.3 \%$ normal weight, $40.6 \%$ overweight, and $33.8 \%$ obese. Underweight was associated with increased suicide risk (adjusted hazard ratio $[A H R]=1.17,95 \%$ $\mathrm{Cl}: 1.01,1.36)$ compared to normal. Overweight and obese status were associated with lower risk $(\mathrm{AHR}=0.78,95 \% \mathrm{Cl}: 0.74,0.82 ; \mathrm{AHR}=0.63,95 \% \mathrm{Cl}: 0.60,0.66$, respectively). Among suicide decedents, high lethality methods were most common among underweight and least common among obese individuals. Adjusting for covariates, BMI was not associated with method lethality, yet some associations were observed between BMI and specific methods.

Conclusion: Among VHA patients, BMI was negatively associated with suicide risks. These differences may partly relate to choice of suicide method. Low BMI offers an additional resource for clinical suicide risk assessments.

Obesity (2014) 21, 269-276. doi:10.1002/oby.20422

\section{Introduction}

Suicide prevention is a national priority. In 2007, researchers reported a negative association between Body Mass Index (BMI) and suicide risks in Veterans: Among male Veteran respondents to the National Health Interview Survey, overweight individuals had lower suicide risks than those with normal weight (1). This article reports analyses of associations between BMI and suicide among over four million patients in the Veterans Affairs health system, the Veterans Health Administration (VHA).

In the United States, $72 \%$ of men and $64 \%$ of women are either overweight or obese (2). BMI is associated with differential morbidity and mortality. Being overweight or obese is associated with increased risk of chronic illnesses and greater mortality from diabetes, cardiovascular disease (CVD), and some cancers. Being underweight is associated with greater mortality from noncancer, non-CVD causes $(3,4)$.

The finding of lower suicide risks among Veterans who are overweight has been considered surprising because obesity has been pos- itively associated with depression and other established suicide risk factors $(5,6)$. However, the literature regarding BMI and depression is inconsistent (7-12). Likewise, findings regarding BMI and suicide attempts and ideation are variable (13).

Yet a growing and consistent literature supports the observation of lower suicide risks among individuals with elevated BMI (14-18). For example, adjusting for psychological, social, and lifestyle measures, a study of 74,332 adults in Norway observed that elevated BMI was associated with reduced suicide risk, even while simultaneously being associated with greater likelihood of depression (19). With few exceptions (20), the literature presents a pattern that, as noted by Mukamal and Miller, goes "well beyond any possibility of chance alone" (21).

Several hypotheses have been developed to explain the association. For example, researchers have proposed links between correlates of low BMI and serotonin deficiency, which is itself a correlate of suicidality (22). It has been suggested that elevated BMI is a marker

\footnotetext{
${ }^{1}$ US Department of Veterans Affairs (VA), Office of Mental Health Operations (OMHO), Washington, District of Columbia, USA. Correspondence: John F. McCarthy (john.mccarthy2@va.gov) ${ }^{2}$ VA OMHO Serious Mental Illness Treatment Resource and Evaluation Center, Ann Arbor, Michigan, USA ${ }^{3}$ VA Center for Clinical Management Research, Washington, District of Columbia, USA ${ }^{4}$ Department of Psychiatry, University of Michigan, Ann Arbor, Michigan, USA

Disclosures: The authors have no competing interests.

Author Contributions: All authors contributed to the conception and design of the study. Dr. McCarthy drafted the manuscript. All authors reviewed and provided critical input and approved the final manuscript. Ms. Austin conducted study data management and analyses.

This article is a US government work and, as such, is in the public domain in the United States of America.

Received: 8 December 2012 Accepted: 5 February 2013 Published online 20 March 2013. doi:10.1002/oby.20422
} 
for insulin resistance, higher levels of free fatty acids, and greater tryptophan, leading to higher serotonin levels and reduced suicide (23). However, an inverse association between BMI and suicide has been observed even when controlling for dietary fatty acids (16). Alternatively, low cholesterol may increase suicide risks via reductions in central serotonergic activity (24). This research evidence is limited and inconsistent, however $(17,20,25)$. Other hypotheses link personality factors to underweight and overweight status, noting that high neuroticism is associated with underweight status and low conscientiousness with obese status (26). Neuroticism is associated with increased risk of major depression, largely because it "indexes the genetic risk for depressive illness" (27). Despite considerable activity, the puzzling inverse association between BMI and suicide risk has not been explained.

In response, Mukamal and Miller examined associations between BMI and diverse suicide risk factors, using data from the Behavioral Risk Factor Surveillance System (28). They did not identify suicide risk factors that were consistently inversely related to BMI. They concluded that these factors may be unlikely mediators of the inverse association between BMI and suicide risks and that greater BMI "may act 'downstream' of suicidal ideation or attempts in preventing suicide." They call for studies of the mechanisms underlying the BMI-suicide association, to inform suicide prevention by influencing risk factors "late in the causal chain."

Suicide mortality patterns are influenced by not only those factors influencing suicide attempts but also those affecting choice of method (29). Differential choice of method of suicide has been proposed as a factor affecting BMI-related suicide mortality. For example, suicide risks among individuals with anorexia nervosa and bulimia nervosa are greater than those in the general population, and individuals with eating disorders have greater determination and resistance to pain and less fear of death, resulting in their choosing more lethal means when attempting suicide (30). Similar mechanisms may apply with regard to the method of suicide across BMI categories. It is important, therefore, to examine associations between method of suicide and BMI.

Although the literature includes well-designed large populationbased cohort studies, these have had some limitations that are not present in the VHA context. The largest integrated health system in the United States, the VHA electronic medical record system enables systematic assessment of individual risk factors, including diagnoses for psychiatric and non-psychiatric conditions. Vital status and cause of death information from the National Death Index (NDI) are routinely searched for VHA user cohorts (31). Further, VHA records include anthropometric measures assessed at various ages.

In this study, we assess associations between BMI and suicide risk, adjusting for sociodemographic and clinical characteristics. Among suicide decedents, we assess associations between BMI and suicide method. We hypothesized that BMI is inversely associated with suicide risks and that BMI is negatively associated with lethality of suicide method.

\section{Methods}

\section{Data}

Using data from the VHA's National Patient Care Database (NPCD), the cohort was drawn from all 5,043,060 individuals with inpatient or outpatient service encounters in any VHA facility during fiscal years (FYs) 2001 or 2002 and who were alive at the start of FY2002 (10/1/01). Vital status and cause of death were derived from NDI search results. The NDI includes date and causes of death per death certificates filed in state vital statistics offices. It is considered the gold standard for mortality assessment sources. Search results were assessed for potential matches and "true matches" were identified using established procedures. Data regarding patient height and weight were derived from the VHA national Corporate Data Warehouse (CDW). Evaluation of these CDW data indicates close concordance with records from VHA regional data sources (32).

\section{Measures}

Indicators of age and sex were drawn from the NPCD. Age at the start of FY2002 was categorized as 18-29, 30-64, or 65+. Information regarding race/ethnicity was not consistently available and therefore was not included. We assessed diagnoses in FY2001 for patients with use in that year, otherwise in FY2002. Diagnoses included substance abuse/dependence, bipolar disorder, major depressive disorder, other depression diagnoses, post-traumatic stress disorder (PTSD), other anxiety disorders, and schizophrenia. We included indicators of nicotine dependence and eating disorders (anorexia nervosa or bulimia nervosa) and nine medical conditions that varied most substantially across the BMI weight categories (chronic obstructive pulmonary disease [COPD], head cancer, nicotine dependence, hypertension, diabetes, autoimmune disease, dementia, alcohol withdrawal delirium, and hemi-/paraplegia). We constructed an indicator of receipt of mental health encounters during FY2001 or, for patients with no services that year, in FY2002. Also, we identified the facility where patients had their last utilization in the period FY2001-2002 and the corresponding VHA regional network.

Dates and causes of death were identified from the NDI data. Suicides were indicated by International Classification of Diseases, Tenth Revision, codes X60-X84, and Y87.0. Method of suicide was categorized in two ways: First, we assessed the following causes: firearms, poisoning, suffocation, and other methods. Second, we categorized the causes in terms of higher lethality (involving hanging, suffocation, or strangulation [X70]; drowning [X71]; use of firearms [X72-X74]; other causes [X75-X82, Y870]) versus lower lethality (poisoning [X60-X69] and other methods [X83-X84]).(21)

Values for height and weight were searched for the period from FY2000 to 2003, and measures that were most concurrent with the patient's first VHA use in the period FY2001-2002 were selected. Twenty percent were excluded, because of missing values for height or weight. BMI was calculated in metric units by multiplying a conversion factor of $703 \times$ (weight in pounds/(height in inches $\times$ height in inches)). A total of 16,890 patients $(0.3 \%)$ were excluded because of implausible values that may reflect data entry problems (height less than 48 inches or greater than 84 inches; weight less than 75 pounds or greater than 500 pounds) (33). We excluded 22 individuals who met these criteria yet had implausible values that were identical for height in inches and weight in pounds (32). BMI was categorized as underweight $(<18.5)$, normal (18.5-24.9), overweight (25-29.9), or obese (BMI $\geq 30$ ). The study cohort included 4,005,640 patients.

This project was reviewed and approved by a VHA human subjects committee. 


\section{Analyses}

Descriptive statistics were calculated for the study cohort, overall, by BMI category, and by suicide status. We used chi-squared tests to compare measures by BMI category and suicide status. We conducted similar analyses specific to suicide decedents. Also, we compared available data for individuals who were excluded from versus retained in study analyses.

Using proportional hazards regression, we evaluated associations between BMI categories and suicide mortality, controlling for age, sex, psychiatric and medical diagnosis categories, nicotine dependence, eating disorders, mental health services receipt, and VA regional network. The model included covariance sandwich estimators to adjust for the clustering of individuals within VA facilities (specified by three-digit facility prefix codes.) Risk time for an observed suicide death began on the first day of FY2002 for those with VHA utilization in FY2001, and on the date of first utilization in FY2002 for those without VHA utilization in FY2001. Risk periods ended either at death or at the end of FY2009, whichever came first.

Among suicide decedents, descriptive statistics were assessed regarding BMI and suicide methods, overall and by sex and age groups. Multivariable Generalized Estimating Equations (GEE) regression analyses assessed associations between BMI categories and use of high lethality means. An initial model adjusted only for BMI categories and VHA regional network. A second model added sociodemographic measures and the final model added the remaining study covariates. Likewise, we assessed predictors of use of specific categories of methods, as compared to all others. These included use of firearms, poisoning, and strangulation.

Study analyses were conducted using SAS (version 9; SAS Institute, Cary, NC, USA)

\section{Results}

Study analyses indicated that $1.4 \%$ of VHA patients in the study cohort were categorized as underweight, $24.3 \%$ normal weight, $40.6 \%$ overweight, and 33.8\% obese. Between FY2002 and 2009, there were 10,169 suicide deaths in the study cohort. Table 1 presents information regarding patient characteristics, overall and by body mass categories. For each measure, there were significant differences across BMI categories. Individuals who were overweight or obese were somewhat less likely to be female than those with normal or underweight status. Mean age was lowest among underweight individuals. Underweight was associated with greater prevalence of diagnosed substance use disorder, non-MDD depression, non-PTSD anxiety, and schizophrenia. Overweight and obese individuals had greater prevalence of bipolar disorder, major depressive disorder, and PTSD. Underweight individuals had greater prevalence of COPD, dementia, alcohol withdrawal delirium, hemi- or paraplegia, and eating disorders. Obese individuals had greater prevalence of hypertension, diabetes, and autoimmune disease. Nicotine dependence was less prevalent among overweight and obese individuals. Receipt of mental health treatment was more prevalent among individuals with underweight and those with obesity. The prevalence of mortality from any cause was highest among individuals who were underweight and lowest among those who were obese. The prevalence of suicide was lower among individuals with obesity than in other body mass categories.

In bivariate analyses, suicide decedents were significantly more likely to be underweight (1.7\% vs. $1.3 \%)$ and normal weight $(30.9 \%$ vs. $24.2 \%)$ and less likely to be obese (26.9\% vs. $33.8 \%)$ than patients who did not die from suicide. They were also more likely to be male, between 30 and 64 years old, to have psychiatric conditions, to have received mental health treatment, and to have had COPD, head cancer, nicotine dependence, hemi- or paraplegia, and delirium. Also, they were less likely to have had hypertension, diabetes, cancer, and autoimmune disease.

Table 2 presents results from multivariable proportional hazards regression analyses. Adjusting for model covariates, individuals who were underweight had greater risks of suicide, relative to those with normal weight (adjusted hazard ratio 1.17, 95\% confidence interval [CI] 1.01, 1.36), and those who were overweight or obese had lower risks of suicide (adjusted hazard ratio 0.78 [0.74, 0.82] and 0.63 $[0.60,0.66]$, respectively). Other risk factors for suicide included male sex, older age, each of the psychiatric conditions, receipt of mental health treatment, eating disorders, COPD, head cancer, nicotine dependence, and alcohol withdrawal delirium. Hypertension and diabetes were associated with lower risks.

Table 3 presents information regarding patient characteristics and method of suicide, overall and by body mass category, among suicide decedents. The prevalence of suicide risk factors was greater among suicide decedents than in the overall patient population. The prevalence of risk factors varied by BMI category for many variables. This generally followed the pattern observed for the overall VHA population, with the exception that the among suicide decedents the prevalence of schizophrenia was greater among those who were obese than those who were underweight.

Method of suicide varied significantly across body mass categories. Prevalence of the use of firearms was most common among those who were underweight. Poisoning was most prevalent among patients who were obese. Suffocation was most prevalent among patients with normal weight. Similar patterns were observed in analyses that were limited to men. Method of suicide did not differ significantly by body mass category when analyses were restricted to women, individuals aged 18-29, and individuals aged 65 and older. Similar trends were also observed among individuals aged 30-64, although the differences in prevalence were smaller than in the analysis overall.

Lethality of method differed significantly by BMI category. Suicide by methods of high lethality was most prevalent among underweight suicide decedents and lowest among those who were obese. Conversely, methods of lower lethality were most common among decedents who were obese than those who were underweight. We note that in subanalyses stratified by sex, we found that among men, methods differed significantly, with a larger observed differential between underweight and obese decedents. We did not observe significant differences among women. When stratified by age group, method differed significantly by BMI category among those aged 30-64, with greatest use of low lethality methods among those who were obese. In sensitivity analyses, we did not observe significant interactions between BMI category and age groups.

GEE analyses of predictors of use of high lethality means are presented in Table 4. In the initial model, obese status was associated with decreased likelihood of use of high lethality means, relative to normal $(\mathrm{OR}=0.83 ; 95 \% \mathrm{C} \pm 0.70,0.98)$. This association became nonsignificant when adjusting for sociodemographic measures. In the full model, BMI categories were not associated with use of high lethality means. Use of high lethality means was more likely among suicide decedents who were male, at least 65 years old, in rural 
TABLE 1 Patient characteristics, by body mass category

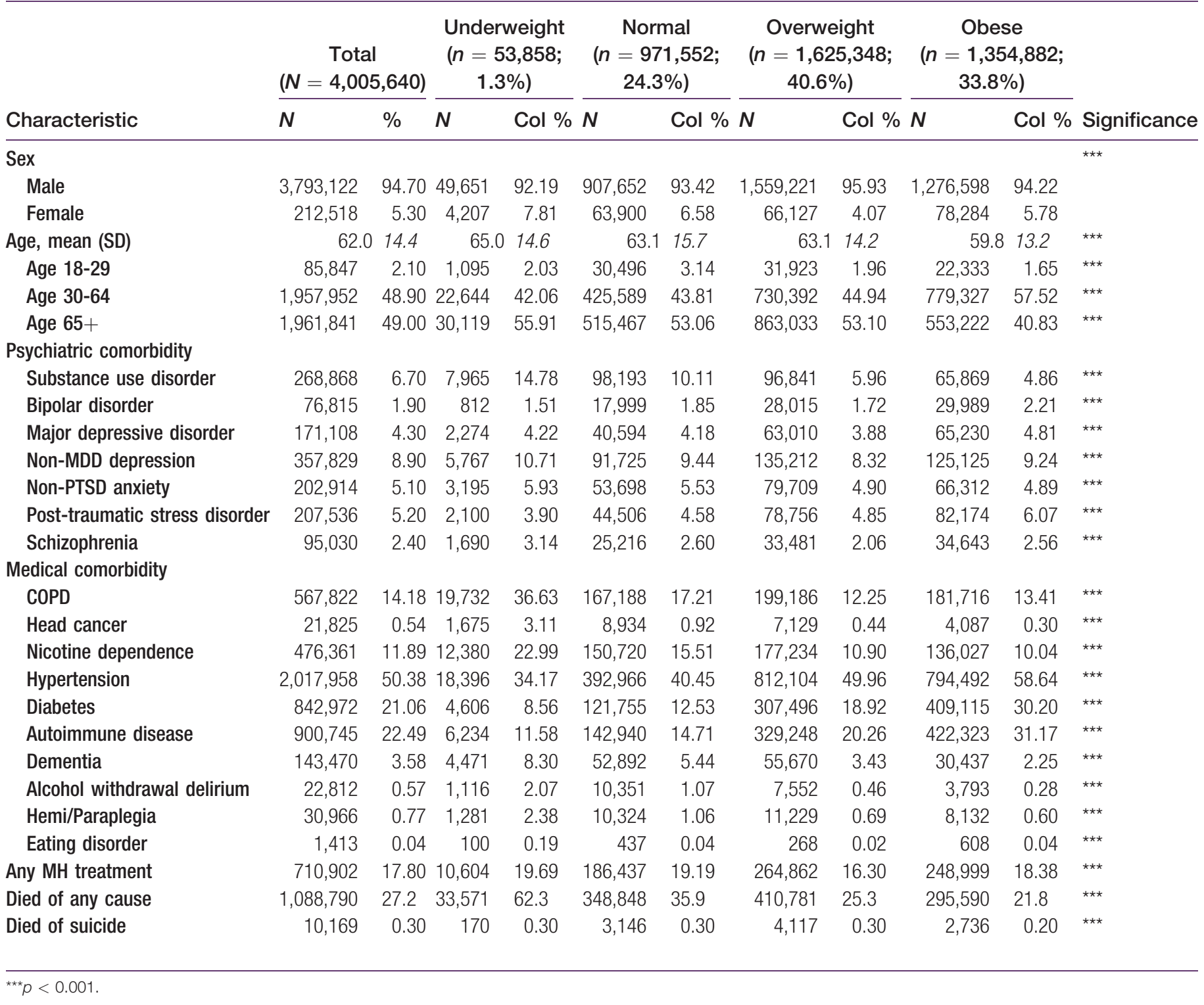

areas, with diabetes, and dementia. It was less likely among those with bipolar disorder, schizophrenia, depression, PTSD, substance use disorder, and autoimmune disease.

Supplemental analyses, adjusting for all covariates, indicated that underweight individuals were more likely than normal weight individuals to use firearms than other methods $(\mathrm{OR}=1.78,95 \% \mathrm{CI} 1.22$, 2.58). Also, compared to normal weight, being underweight was associated with decreased likelihood of strangulation as a means of suicide $(\mathrm{OR}=0.52,95 \%$ CI $0.29,0.94)$, as was being overweight $(\mathrm{OR}=0.87$, $95 \%$ CI $0.75,1.00)$ and obese $(\mathrm{OR}=0.74,95 \%$ CI $0.62,0.88)$.

\section{Discussion}

A substantial challenge for suicide prevention is recognition of risk factors. Some indicators of elevated risk may be salient, such as prior attempts and depression. Others may be less well recognized and, consequently, elevated risk may go unidentified. For example, many suicide decedents have had no recent contact with mental health professionals (34), yet nearly $90 \%$ had diagnosable psychiatric conditions (35). Analysis of factors potentially associated with suicide mortality may advance understanding of suicide, enhance risk assessments, and guide prevention initiatives.

This study sought to explore a finding among Veterans that was regarded as surprising (1). That study has since been replicated, including the entire National Health Interview sample (36). The observation also has been repeatedly documented in large longitudinal analyses with various study designs and populations. Although both biological and behavioral explanations have been advanced and developed, the evidence for these mechanisms has been contradictory.

This study makes three contributions. First, we assess BMI as a predictor of suicide among more than four million individuals receiving 
TABLE 2 Multivariable proportional hazards regression results $(N=4,005,662)$

\begin{tabular}{|c|c|c|c|c|}
\hline & $\begin{array}{l}\text { Hazard } \\
\text { ratio }\end{array}$ & $\begin{array}{l}\text { Lower } \\
\text { bound } \\
95 \% \mathrm{Cl}\end{array}$ & $\begin{array}{l}\text { Upper } \\
\text { bound } \\
95 \% \mathrm{Cl}\end{array}$ & $P$-value \\
\hline \multicolumn{5}{|l|}{$\mathrm{BMI}$} \\
\hline Underweight & 1.17 & 1.01 & 1.36 & 0.0418 \\
\hline Normal & - & - & - & - \\
\hline Overweight & 0.78 & 0.74 & 0.82 & $<0.0001$ \\
\hline Obese & 0.63 & 0.60 & 0.66 & $<0.0001$ \\
\hline \multicolumn{5}{|l|}{ Sex } \\
\hline Male & 2.74 & 2.40 & 3.12 & $<0.0001$ \\
\hline Female & - & - & - & - \\
\hline \multicolumn{5}{|l|}{ Age } \\
\hline age $18-29$ & 0.80 & 0.69 & 0.92 & 0.002 \\
\hline age $30-64$ & 0.85 & 0.80 & 0.90 & $<0.0001$ \\
\hline age $65+$ & - & - & - & - \\
\hline \multicolumn{5}{|l|}{ Psychiatric comorbidity } \\
\hline Substance use disorder & 1.34 & 1.24 & 1.45 & $<0.0001$ \\
\hline Bipolar disorder & 1.98 & 1.81 & 2.16 & $<0.0001$ \\
\hline Major depressive dis. & 1.89 & 1.75 & 2.05 & $<0.0001$ \\
\hline Non-MDD depression & 1.68 & 1.57 & 1.81 & $<0.0001$ \\
\hline Non-PTSD anxiety & 1.34 & 1.24 & 1.44 & $<0.0001$ \\
\hline Post-traumatic stress dis. & 0.89 & 0.82 & 0.97 & 0.01 \\
\hline Schizophrenia & 1.30 & 1.18 & 1.44 & $<0.0001$ \\
\hline Any VHA MH treatment & 1.33 & 1.24 & 1.43 & $<0.0001$ \\
\hline \multicolumn{5}{|l|}{ Medical comorbidity } \\
\hline Eating disorder & 1.07 & 0.53 & 2.18 & 0.8493 \\
\hline Chronic obstr. pul. dis. & 1.28 & 1.22 & 1.35 & $<0.0001$ \\
\hline Head cancer & 1.69 & 1.35 & 2.11 & $<0.0001$ \\
\hline Nicotine dependence & 1.15 & 1.09 & 1.21 & $<0.0001$ \\
\hline Hypertension & 0.88 & 0.84 & 0.91 & $<0.0001$ \\
\hline Diabetes & 0.77 & 0.66 & 0.89 & 0.0006 \\
\hline Autoimmune disease ${ }^{\star *}$ & 1.15 & 1.00 & 1.34 & 0.0569 \\
\hline Dementia & 0.91 & 0.80 & 1.03 & 0.1418 \\
\hline Alcohol withdrawal delirium & 1.33 & 1.12 & 1.59 & 0.0015 \\
\hline Hemi/Paraplegia & 1.19 & 0.97 & 1.46 & 0.0934 \\
\hline
\end{tabular}

"-" indicates reference category.

Cox proportional hazards survival model adjusting for clustering at facility level and listed covariates and VHA regional network.

PTSD, post-traumatic stress disorder; COPD, chronic obstructive pulmonary disorder.

care in the VHA health system, followed for up to 8 years. Study findings indicate increased suicide risk among individuals who are underweight, relative to those of normal weight, and decreased risk among those categorized as overweight or obese. Second, this study design offers important improvements compared to previous studies. We adjusted for patient characteristics that included a range of clinical condition indicators derived from diagnosis data from the VHA's well-regarded electronic medical record system. Third, to our knowledge, this was the first study to examine associations between BMI and method of suicide among suicide decedents. Study findings were suggestive but inconclusive regarding whether something "downstream of attempts" affects suicide mortality (28). In bivariate analyses, we observed that method of suicide differed by BMI status, with greater use of more lethal methods among those with lower BMI and, conversely, greater use of less lethal methods (i.e., poisoning) among those with higher BMI. Although this association with use of high lethality means was nonsignificant in the full model, underweight BMI was associated with increased odds of use of firearms by suicide decedents. Also, strangulation was more likely among those with normal weight than those who were either underweight, or overweight or obese.

We evaluated whether, among suicide decedents, method of suicide differed by BMI status. Our hypothesis was that differential use of more lethal means accounts for the frequently observed negative association between BMI and suicide. We hypothesized that among individuals who attempt suicide, those with greater BMI are more likely than those with lower BMI to use methods with lower lethality, such as poisoning (37), and that they are less likely to use higher lethality means, such as firearms. Bivariate analyses did identify differences in suicide methods, among suicide decedents, by BMI status. For example, $16.8 \%$ of suicides among obese patients involved poisoning, versus $9.4 \%$ among underweight individuals; that is, use of poisoning was $79 \%$ greater among obese individuals than among underweight individuals. By comparison, the sex differential in use of poisoning was larger: $47.9 \%$ of female suicides involved poisoning, versus $13.6 \%$ of male suicides (252\% greater). In the multivariable analysis, BMI was not a significant predictor of use of higher versus lower lethality means. However, as noted above, BMI was significant in some method-specific analyses. We conclude that although associations between BMI and methods of suicide may partly underlie the negative association between BMI and suicide risk, it is unlikely that this puzzling association may be explained simply by differential lethality of suicide methods.

Further, we note that associations between BMI and method of suicide may not be explained by differential access to lethal means. Among male respondents to the Behavioral Risk Factor Surveillance System, those who were overweight or obese were more likely than those with normal BMI scores to have a firearm at home and to have a loaded firearm at home (28). This suggests that there are important factors affecting suicide behavior despite differential access to lethal means.

We note several limitations. First, analyses excluded individuals for whom height or weight information were either unavailable or were implausible. Those who were excluded were more likely to be female, younger, and less likely to have psychiatric diagnoses. Second, although the analyses adjusted for VHA regional network and for clustering at the facility level, we did not include rural/urban status as a model covariate (38) in the proportional hazards regression model, as this would result in excluding $3 \%$ of VHA users. In sensitivity analyses restricted to individuals with complete data, including rural/urban status, the pattern of results did not differ. Third, the available data regarding height and weight are subject to measurement error. In addition, measurement may be more complicated and hence more likely to be missing or inaccurate for specific patient subpopulations, such as obese patients, amputees, and individuals who are wheelchair bound (32). Further, we note that suicide patterns among VHA patients may not be generalizable to other populations. For example, firearm suicide rates and firearm use among suicide decedents may be greater among Veterans than among non- 


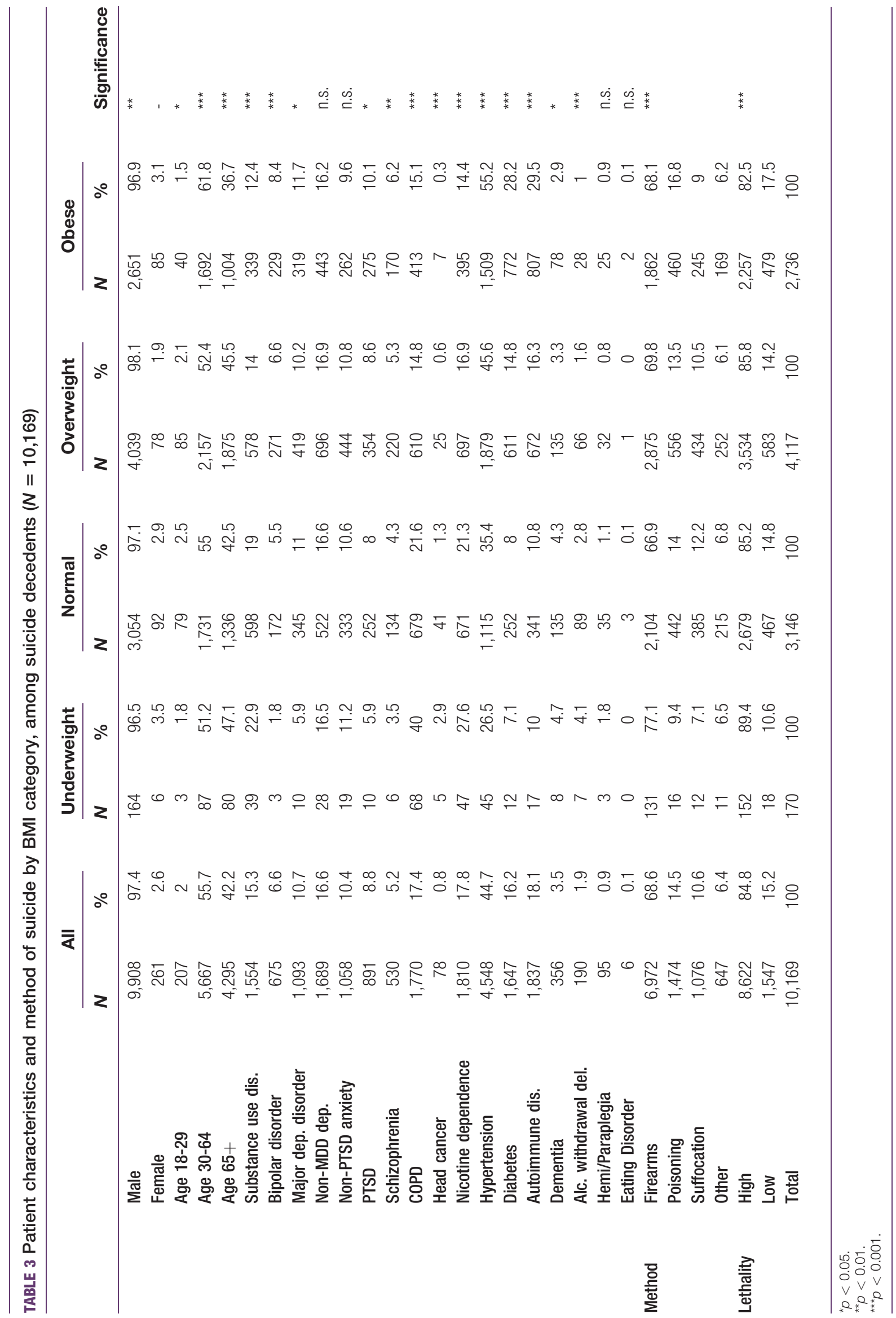




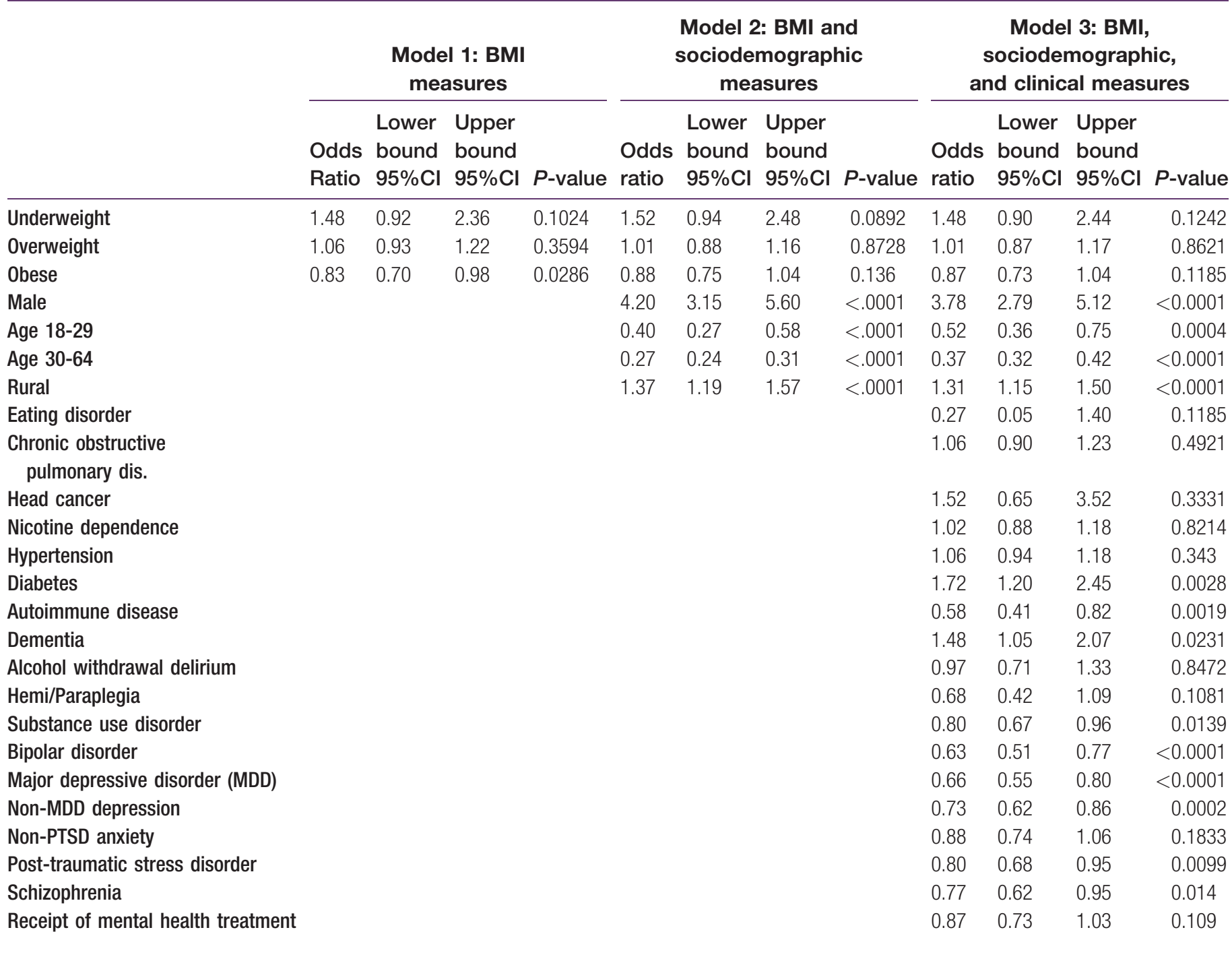

*Reference categories: Normal BMI, female sex, age 65+, urban. Includes adjustment for VA regional network.

Veterans (39). Finally, despite adjustment for demographics, mental health treatment, and a range of diagnosed conditions, there may be important unmeasured confounding factors. In particular, these may relate to risk factors for all-cause mortality, which was substantially inversely associated with BMI categories.

Future studies should explicate BMI-related differentials in methods of suicide, both among Veterans and non-Veterans. In particular, studies should evaluate potential differentials with regard to suicide attempts. The VHA is actively studying suicide mortality among VHA patients and all Veterans (40). This study adds to a growing body of research studies documenting negative associations between BMI and suicide risk. We extend the literature to include VHA patients and to include indicators of a range of clinical diagnosis indicators. Further, we document limited associations between BMI and method of suicide. This study confirms a negative association between BMI and suicide risk. This provides an additional resource for providers when evaluating patient suicide risks, even adjusting for measures of psychiatric and nonpsychiatric morbidity. Findings inform ongoing VHA suicide prevention activities. $\mathrm{O}$

(C) 2013 The Obesity Society

\section{References}

1. Kaplan MS, Huguet N, McFarland BH, Newsom JT. Suicide among male Veterans: A prospective population-based study. Epidemiol J Community Health 2007;61: 619-624.

2. Flegal KM, Carroll MD, Ogden CL, Curtin LR. Prevalence and trends in obesity among US adults, 1999-2008. JAMA 2010;303:235-241.

3. Kress AM, Hartzel MC, Peterson MR. Burden of disease associated with overweight and obesity among US military retirees and their dependents, aged 38-64, 2003. Prev Med 2005;41:63-69.

4. Must A, Spadano J, Coakley EH, Field AE, Colditz G, Dietz WH. The disease burden associated with overweight and obesity. JAMA. 1999;282:1523-1529.

5. Simon GE, Von Korff M, Saunders K, et al. Association between obesity and psychiatric disorders in the US adult population. Arch Gen Psychiatry 2006;63: 824-830. 
6. Ilgen MA, Bohnert ASB, Ignacio RV, et al. Psychiatric diagnoses and risk of suicide in Veterans. Arch Gen Psychiatry 2010;67:1152-1158.

7. Friedman MA, Brownell KD. Psychological correlates of obesity: moving to the next generation. Psychol Bull 1995;117:3-20.

8. Barry D, Pietrzak RH, Petry NM. Gender differences in associations between body mass index and DSM-IV mood and anxiety disorders: results from the national epidemiological survey on alcohol and related conditions. Ann Epidemiol 2008;18: 458-466.

9. Carpenter KM, Hasin DS, Allison DB, Faith MS. Relationship between obesity and DSM-IV major depressive disorder, suicide ideation, and suicide attempts: results from a general population study. Am J Public Health 2000;90:251-257.

10. Palinkas LA, Wingard DL, Barrett-Connor E. Depressive symptoms in overweight and obese older adults: a test of the "Jolly Fat" hypothesis. J Psychosom Res 1996; 40:59-66.

11. Kress AM, Peterson MR, Hartzell MC. Association between obesity and depressive symptoms among U.S. military active duty personnel, 2002. J Psychosom Res 2006; 60:263-271.

12. de Wit L, Luppino F, van Straten A, Penninx B, Zitman F, Cuijpers P. Depression and obesity: a meta-analysis of community-based studies. Psychiatry Res 2010;178: 230-235.

13. Batty GD, Whitley E, Kivimaki M, Tynelius P, Rasmussen F. Body mass index and attempted suicide: Cohort study of 1,133,019 Swedish men. Am J Epidemiol 2010; 172:890-899.

14. Paffenbarger RS, Asnes DP. Chronic disease in former college students: III. Precursors of suicide in early and middle Life. Am J Public Health 1966;56:1026-1036.

15. Magnusson PKE, Rasmussen F, Lawlor DA, Tynelius P, Gunnell D. Association of body mass index with suicide mortality: a prospective cohort study of more than one million men. Am J Epidemiol 2006;163:1-8.

16. Mukamal KJ, Kawachi I, Miller M, Rimm EB. Body mass index and risk of suicide among men. Arch Intern Med 2007;167:468-475.

17. Jee SH, Kivimaki M, Kang HC, Park IS, Samet JM, Batty GD. Cardiovascular disease risk factors in relation to suicide mortality in Asia: prospective cohort study of over one million Korean men and women. Eur Heart J 2011;229:1-8.

18. Mukamal KJ, Rimm EB, Kawachi I, O’Reilly EJ, Calle EE, Miller M. Body mass index and risk of suicide among one million US adults. Epidemiology 2010;21: 82-86.

19. Bjerkeset O, Romundstad P, Evans J, Gunnell D. Association of adult body mass index and height with anxiety, depression, and suicide in the general population. Am J Epidemiol 2008;167:193-202.

20. Chang SS, Wen CP, Tsai MK, Lawlor DA, Yang YC, Gunnell D. Adiposity, its related biologic risk factors, and suicide: a cohort study of 542,088 Taiwanese adults. Am J Epidemiol 2012;175:804-815.

21. Mukamal KJ, Miller M. Invited commentary: body mass index and suicide untangling an unlikely association. Am J Epidemiol 2010;172:900-904.

22. Mann JJ, McBride A, Brown RP, et al. Relationship between central and peripheral serotonin indexes in depressed and suicidal psychiatric inpatients. Arch Gen Psychiatry 1992;49:442-446.

23. Golomb BA, Mednick SA, Tenkanen L. Suicide: a weighty matter? Arch Intern Med 2007; 167:1908.

24. Asellus P, Nordstrom P, Jokinen J. Cholesterol and CSF 5-HIAA in attempted suicide. J Affect Disord 2010;125:388-392.
25. de Leon J, Mallory P, Maw L, Susce MT, Perez-Rodriguez MM, Baca-Garcia E. Lack of replication of the association of low serum cholesterol and attempted suicide in another country raises more questions. Ann Clin Psychiatry 2011;23: 163-170.

26. Terracciano A, Sutin AR, McCrae RR, et al. Facets of personality linked to underweight and overweight. Psychosom Med 2009;71:682-689.

27. Kendler KS, Gatz M, Gardner CO, Pedersen NL. Personality and major depression: a Swedish longitudinal, population-based twin study. Arch Gen Psychiatry 2006;63: 1113-1120.

28. Mukamal KJ, Miller M. BMI and risk factors for suicide: Why is BMI inversely related to suicide? Obesity 2009;17:532-538.

29. Miller M, Azrael D, Hemenway D. The epidemiology of case fatality rates for suicide in the northeast. Ann Emerg Med 2004;43:723-730.

30. Holm-Denoma JM, Witte TK, Gordon KH, et al. Deaths by suicide among individuals with anorexia as arbiters between competing explanations of the anorexia-suicide link. J Affect Disord 2008;107:231-236.

31. McCarthy JF, Valenstein M, Kim HM, et al. Suicide mortality among patients receiving care in the Veterans Health Administration Health System. Am J Epidemiol 2009;169:1033-1038.

32. Noel PH, Copeland LA, Perrin RA, et al. VHA corporate data warehouse height and weight data: opportunities and challenges for health services research. $J$ Rehabil Res Dev 2010;47:739-750.

33. Das SR, Kinsinger LS, Yancy WS, et al. Obesity prevalence among Veterans at Veterans Affairs Medical Facilities. Am J Prev Med 2005;28:291-294.

34. Basham C, Denneson LM, Millet L, Shen X, Duckart J, Dobscha SK. Characteristics of VA Health Care Utilization of U.S. Veterans who completed suicide in Oregon between 2000 and 2005. Suicide Life Threat Behav 2011;41:287-296.

35. Conner KR, Duberstein PR, Conwell Y, Seidlitz L, Caine ED. Psychological vulnerability to completed suicide: a review of empirical studies. Suicide Life Threat Behav 2001;31:367-385.

36. Kaplan MS, McFarland BH, Huguet N. The relationship of body weight to suicide risk among men and women: Results from the US National Health Interview Survey linked mortality file. J Nerv Ment Dis 2007;195:948-951.

37. Centers for Disease Control and Prevention. National suicide statistics at a Glance: case fatality rate among persons ages 10 years and older for males and females separately, and by selected mechanism for both sexes combined, United States, 2005-2009. http://www.cdc.gov/violenceprevention/suicide/statistics/case fatality.html (Accessed 12/28/12).

38. McCarthy JF, Blow FC, Ignacio RV, Ilgen MA, Austin KL, Valenstein M. Suicide among patients in the Veterans Affairs Health System: rural-urban differences in rates, risks, and methods. Am J Public Health 2012;102:S111-S117.

39. Kaplan MS, McFarland BH, Huguet N. Firearm suicide among Veterans in the general population: findings from the National Violent Death Reporting System. $J$ Trauma 2009;67:503-507.

40. Katz IR, McCarthy JF, Ignacio RV, Kemp J. Suicide among Veterans in 16 states, 2005 to 2008: comparisons between utilizers and nonutilizers of Veterans Health Administration (VHA) services based on data from the National Death Index, the National Violent Death Reporting System, and VHA Administrative Records. Am J Public Health 2012;102:S105-S110. 